\title{
RNA interference on target
}

It has only been 5 years since Tom Tuschl and colleagues showed that the RNA interference (RNAi) pathway was functional in cultured mammalian cells, and that it was possible to trigger this process to specifically silence a gene using a small interfering RNA (siRNA). This discovery was one of these rare methodological leaps that revolutionize a field. Lossof-function analysis, the geneticist's good old trick of stopping the expression of a gene to discover its function, was now possible in mammalian cells with unrivaled efficiency and ease. Nature Methods celebrates this anniversary by dedicating a Focus to RNA interference in mammalian cells.

On this occasion, five years after the seminal experiment, Tuschl and Yi Pei contribute a Perspective entitled "On the art of identifying effective and specific siRNAs” (p. 670)—unfortunately, achieving efficiency and specificity is still very much an art. In fact, siRNA design relies on rules based on an incomplete understanding of the molecular mechanism blended with empirical observations. Indeed, despite phenomenal progress, a lot remains to be understood about the RNAi process as it occurs naturally or in response to an investigator's intervention. Under such circumstances, it helps to keep in mind that RNAi is still an immature toolextremely successful, but immature.

Maturation will most likely come from an iterative process by which the experimental performance of an initial set of RNAi triggers, designed according to the present knowledge, guides the refinement of the design rules, which in turn will sometimes lead to mechanical insights. Another exciting possibility comes from the development of methods to generate sets of siRNAs or short-hairpin RNAs (shRNAs) that do not rely on design rules-such as those reviewed by Frank Buchholz and colleagues (p. 696) - and which could thus provide unbiased insight and new design rules.

In the meantime, however, it is important to proceed with caution. Inevitably, the use of such an immature tool has already led to the publication of erroneous knockdown results. In the wake of the original enthusiasm, the so-called 'off-target effects' have been a humbling discovery. It is clear now that the RNAi machinery tolerates some sequence mismatches. Genes can be silenced by siRNAs that are only partially complementary to their sequence, and the level of the mismatch tolerance is still under investigation. In addition, more sweeping and less sequence-specific effects, such as induction of the interferon response, engagement of toll-like receptors and saturation of the RNAi machinery, can lead to phenotypes completely unrelated to the silencing of the target.

Because the mechanisms causing off-target effects are only partially understood, the only remedy at this stage is the use of specificity controls. On page 677 , Bryan Cullen reviews the potential pitfalls of RNAi experiments and suggests stringent controls to include when knocking down one's favorite gene in mammalian cells. These recommendations provide a very useful framework for new and seasoned RNAi users to design and analyze their experiments.

Of course it should be noted that different systems, such as non-mammalian model organisms, may have different requirements. It is also important to avoid becoming dogmatic. Even in mammalian cells, it may not always be possible to apply all the controls suggested. Genome-wide RNAi screens, for example, which have been made possible by the development of siRNA and shRNA libraries, present unique logistical challenges when it comes to controlling for offtarget effects. Some of the controls suggested in the case of single-gene knockdown are not conceivable for large-scale screens. However, the major libraries presently available (reviewed in the second part of this Focus) present a degree of gene coverage that should at least allow the confirmation of a phenotype by independent shRNAs. Nevertheless, follow-up validation of the 'hits' of a screen remains critical.

Ultimately, the choice of the appropriate level of control will depend on the question asked-first screen versus definitive phenoptype-and on the amount of additional corroboration that is available from independent experiments. Journal editors certainly have a role in enforcing best practices by ensuring the quality of published RNAi experiments. Some Nature journals have put forward their recommendations, in line with those presented by Cullen in this issue and established following a community discussion (see Nat. Cell Biol. 5, 489; 2003).

The problem, however, keeps evolving, making it difficult to set rules in stone. While our understanding of off-target effects progresses, new reagents are developed and with them come new challenges. A continuing community debate on pitfalls and solutions thus remains necessary. 